\title{
BP-OP-2-7
}

\section{Optimal timing of portal vein embolization (PVE) after preoperative biliary drainage for hilar cholangiocarcinoma}

\author{
Yunghun YOU', Jin Seok HEO', Sang Hyun SHIN², Sung Wook Shin ${ }^{3}$, Hong Suk Park ${ }^{3}$, \\ Kwang Bo Park ${ }^{3}$, Sung Ki $\mathrm{Cho}^{3}$, Dongho Hyun ${ }^{3}$, In Woong HAN*2
}

'Department of Surgery, Eulji University School of Medicine, Daejeon, Korea

${ }^{2}$ Department of Surgery, Samsung Medical Center, Sungkyunkwan University School of Medicine, Seoul, Korea

${ }^{3}$ Department of Radiology and Center for Imaging Science, Samsung Medical Center, Sungkyunkwan University School of Medicine, Seoul, Korea

Introduction: A combination of portal vein embolization (PVE) and preoperative biliary drainage (PBD) in hilar cholangiocarcinoma (CCC) increase the resectability and help to reduce postoperative complications. To date, there is no study on the optimal timing of PVE after PBD in hilar cholangiocarcinoma. The purpose of this study is to identify the optimal timing of PVE after PBD in patients undergoing liver resection for hilar CCC.

Methods: We retrospectively reviewed 64 patients who underwent combined treatment PBD and PVE followed by liver resection for hilar CCC between January 2005 and December 2018 at Samsung Medical Center. The patients were classified into 3 groups: PBDPVE interval ( $\leq 7$ days)-Group1, PBD-PVE interval (8-14 days)-Group 2 and PBD-PVE interval (>14 days)-Group 3. The primary end points were 90 days mortality and posthepatectomy liver failure (PHLF).

Results: There was no significant difference in primary end points between three groups. The PBD-PVE interval showed a marginally significant relationship with Clavien-Dindo grade $\geq 3$ complication $(p=0.053)$ and wound infection $(p=0.099)$. In univariable analysis, risk factors for PHLF were PTBD $(p=0.050)$, Bismuth type IIIb or IV $(p=0.023)$ and ERH or right trisectionectomy $(p=0.045)$. In multivariable analysis, independent risk factors for PHLF was Bismuth type IIIb or IV (HR 4.782, 95\% CI 1.365-16.759; $p=0.014$ ).

Conclusions: Considering that the PBD-PVE interval did not affect PHLF, and the surgical complication increases as the interval increases, it is more beneficial for the patient to performing PVE as early as possible after PBD. This will help to perform the operation faster. 